\title{
Travelling waves, spreading and extinction for Fisher-KPP propagation driven by a line with fast diffusion
}

\author{
Henri BerestyCkI ${ }^{a}$, Jean-Michel Roquejoffre ${ }^{b}$, Luca Rossi $^{a}$ \\ a Ecole des Hautes Etudes en Sciences Sociales, PSL Research University, CNRS \\ Centre d'Analyse et Mathématiques Sociales, \\ 190 - 198 avenue de France F-75244 Paris Cedex 13, France \\ b Institut de Mathématiques de Toulouse, Université Paul Sabatier \\ 118 route de Narbonne, F-31062 Toulouse Cedex 4, France
}

À Juan Luis Vázquez, en signe d'amitié

\begin{abstract}
In an earlier work [9], we introduced a parabolic system to describe biological invasions in the presence of a line (the "road") with a specific diffusion, included in a domain (the "field") subject to Fisher-KPP propagation. We determined the asymptotic spreading speed in the direction of the road, $w^{*}$, in terms of the various parameters. The new result we establish here is the existence of travelling fronts for this system for any speed $c \geqslant w^{*}$. In addition, we show that no front can travel with a speed $c<w^{*}$. We further extend the results to a new model where there is Fisher-KPP growth on the road, embedded in an elsewhere unfavourable plane. This is relevant to discuss certain invasions that have been observed along roads. We establish sharp conditions for extinction/invasion and describe the invasion dynamics.
\end{abstract}

\section{Introduction, presentation of the models}

The goal of this paper is to study the existence of travelling waves to a class of reactiondiffusion systems where the overall propagation is guided by a line having a diffusion on its own. This type of system arises in the description of biological invasions in oriented habitats, where the presence of pathways allows for a more rapid overall movement. For instance, it is observed that the pine processionary moth advances northwards in Europe at a faster pace than anticipated, and the road network system is believed to play a significant role. This is reported in the collective work [23]. The yellow-legged hornet has now colonised the whole South West of France, and it is interesting to examine the maps provided in [17]: it first travelled occurred along the main rivers, 
and from them propagated inland. The invasion of other dangerous insects can be facilitated by road networks, this is believed to be the case for the Aedes Albopictus Mosquito, also referred to as "tiger mosquito", see for instance [2]. Related situations are the movements of animals directed by roads: see for instance the data reported in [21] for the displacements of wolves in the Western Canadian forest. A purely diffusive model has been proposed in [16] to account for the observation of [21]. The propagation of diseases directed by roads is also a related issue, see [24], which makes an interesting parallel with the propagation of rumors.

Let us present the models that will be dealt with in this paper. The first one, introduced in [9], reads:

$$
\left\{\begin{aligned}
\partial_{t} v-d \Delta v & =f(v), & & x \in \mathbb{R}, y>0, t>0, \\
\partial_{t} u-D \partial_{x x} u & =\nu v-\mu u, & & x \in \mathbb{R}, y=0, t>0, \\
-d \partial_{y} v & =\mu u-\nu v, & & x \in \mathbb{R}, y=0, t>0 .
\end{aligned}\right.
$$

The unknowns are the functions $u(t, x)$ and $v(t, x, y)$. The constants $\mu, \nu>0$ and the diffusion coefficients $d, D>0$ are parameters of the model. We assume that $f \in \mathcal{C}^{1}([0,1])$ satisfies the usual Fisher-KPP type condition:

$$
f(0)=f(1)=0, \quad \forall s \in(0,1), \quad 0<f(s) \leqslant f^{\prime}(0) s,
$$

and it is extended to a negative function outside $[0,1]$. This system arises as a description of invasions by a homogeneous reaction and diffusion mechanism of the FisherKPP type in a plane - that we call the field - in the presence of a line, that we call the road. By symmetry, one can restrict the problem to the half-plane $\mathbb{R} \times(0,+\infty)$. The Robin boundary condition for $v$ describes the exchanges between the field and the road: the field yields the amount $\nu v$ to the road, whereas the road yields the amount $\mu u$ to the field.

By now, we have derived several informations about this model. We studied the invasion along the road in [9], showing its dramatic influence. We studied the invasion in the field in [11], revealing that the road had a stronger influence on the overall propagation than expected. In [5], we proved exponential in time invasion on the road in the presence of nonlocal diffusion. In order to check that the last item of Theorem 2.1 persists for other nonlinearities than the logistic one, L. Dietrich [14], [15] constructed travelling waves for (1.1) in a strip, with ignition-type nonlinearity. He proved that their speed grows like $\sqrt{D}$ as $D$ grows to infinity. We refer the reader to the survey [4].

The next setting we wish to study is an extension of system (1.1), which proved to have interesting further effects - see [10]. It is obtained by adding a transport and a mortality term on the road:

$$
\left\{\begin{aligned}
\partial_{t} v-d \Delta v & =f(v), & & x \in \mathbb{R}, y>0, t>0, \\
\partial_{t} u-D \partial_{x x} u+q \partial_{x} u+\rho u & =\nu v-\mu u, & & x \in \mathbb{R}, y=0, t>0, \\
-d \partial_{y} v & =\mu u-\nu v, & & x \in \mathbb{R}, y=0, t>0
\end{aligned}\right.
$$

where $\rho>0$ accounts for a death factor on the road, and $q \partial_{x} u$ is a transport term, that can be of any sign. It is especially relevant to describe situations when the invasive 
species is confronted to external conditions such as water currents, if we think of the line of fast diffusion as a river. Such a situation occurs, for instance, in the diffusion of parasites along rivers, as reported in [18]. The mortality rate on the road may represent adverse conditions on the road.

In this paper we also introduce a new model that shares many common features with the two previous ones, but also reveals interesting qualitative differences. The motivation here is to understand to what extent a hostile field will prevent propagation with growth on the road. The model then reads

$$
\left\{\begin{aligned}
\partial_{t} v-d \Delta v & =-\rho v, & & x \in \mathbb{R}, y>0, t>0, \\
\partial_{t} u-D \partial_{x x} u & =\nu v-\mu u+f(u), & & x \in \mathbb{R}, y=0, t>0 \\
-d \partial_{y} v & =\mu u-\nu v, & & x \in \mathbb{R}, y=0, t>0 .
\end{aligned}\right.
$$

In other words, reproduction occurs on the road, and the field is a purely hostile medium with a death rate $\rho>0$. Similarly to models (1.1) and (1.2), system (1.3) has $(u, v(y)) \equiv(0,0)$ as $1 \mathrm{D}$ steady states. However we will see that it does not always have nontrivial steady states, and this will induce new effects.

Model (1.3) may be relevant to describe the movement of certain parasite weeds, such as the scentless chamomile in Western Canada, which spreads on roads, but which also competes with crops in the surrounding fields. The important agricultural issues posed by this species are described on [13], and a modelling of its spread is proposed in [12]. We will discuss a biological interpretation of certain aspects of our findings after Theorem 2.6 in the next section.

\section{Statement of results}

Previous studies were concerned with the spreading speed for the Cauchy problem, but no results had been derived up to now about travelling waves, except in [14] for the model in a finite strip with a reaction term $f$ of ignition-type. Here we construct travelling waves for (1.1)-(1.3) and obtain a complete characterization of the admissible speeds. For the new model (1.3), we also derive the spreading results for the Cauchy problem.

\subsection{Previous spreading results for Models (1.1) and (1.2)}

In [9], after showing that the unique positive, bounded, stationary solution of the system is $(\nu / \mu, 1)$, we derived the asymptotic speed of spreading in the direction of the road. The main result of that paper is the following.

Theorem $2.1([9])$. (i) There exists $w^{*}=w^{*}(\mu, \nu, d, D)>0$ such that the following is true. Let $(u, v)$ be a solution of (1.1) with a nonnegative, compactly supported initial datum $\left(u_{0}, v_{0}\right) \not \equiv(0,0)$. Then,

- For all $c>w^{*}$, there holds

$$
\lim _{t \rightarrow+\infty} \sup _{|x| \geqslant c t}(u(x, t), v(x, y, t))=(0,0)
$$


- for all $c \in\left[0, w^{*}\right)$, for all $a>0$, there holds

$$
\left.\lim _{t \rightarrow+\infty} \sup _{|x| \leqslant c t, 0 \leqslant y \leqslant a} \mid u(x, t), v(x, y, t)\right)-(\nu / \mu, 1) \mid=0 .
$$

(ii) Let $c_{K}=2 \sqrt{d f^{\prime}(0)}$ denote the Fisher-KPP invasion speed. The following relations hold (for fixed $\mu, \nu$ ):

- If $D \leqslant 2 d$, then $w^{*}(\mu, \nu, d, D)=c_{K}$.

- If $D>2 d$, then $w^{*}(\mu, \nu, d, D)>c_{K}$. Furthermore, $\lim _{D \rightarrow+\infty} \frac{w^{*}(\mu, \nu, d, D)}{\sqrt{D}}$ exists and is a positive real number.

When (2.1)-(2.2) hold for all solutions with nontrivial compactly supported initial datum, then $w^{*}$ is called the (asymptotic) spreading speed in the direction of the road. Roughly speaking, the theorem says that the region of the road (or at finite distance from the road) where the solution is close to the nonzero steady state, and the region of the road where the solution is roughly equal to $(0,0)$ are separated by a boundary moving at velocity $w^{*}$. We refer to [1] for some of the first results of that type. As we will see here, the spreading speed will also turn out to be the minimal speed of travelling waves.

Let us now turn to Model (1.2). The first question is that of steady states. We see that, under the assumptions listed in Section 1, no homogeneous steady states may exist. However, it is proved in [10] that under the extra assumption

$$
s \mapsto f(s) / s \text { is decreasing for } s \in(0,1] \text {, }
$$

Model (1.2) has a unique positive, bounded, steady solution, which additionally is independent of $x$, that we call $\left(u_{s}, v_{s}(y)\right)$. The system for $\left(u_{s}, v_{s}\right)$ reads

$$
\begin{cases}-d v_{s}^{\prime \prime}=f\left(v_{s}\right), & y>0, \\ d v_{s}^{\prime}(0)=\frac{\nu \rho}{\mu+\rho} v_{s}(0), & \end{cases}
$$

and we have $u_{s} \equiv \frac{\nu}{\mu+\rho} v_{s}(0)$. The function $v_{s}$ is increasing and tends to 1 at $+\infty$. For this general system, we obtained a result that is very close to Theorem 2.1, with the difference that the propagation velocity is not the same in the direction of positive $x$ and negative $x$. Moreover, the threshold $D / d>2$ for the spreading speed to be larger than $c_{K}$ is replaced by

$$
\frac{D}{d}>2+\frac{\rho}{f^{\prime}(0)} \mp \frac{q}{\sqrt{d f^{\prime}(0)}}
$$

In this formula, the - sign accounts for the propagation in the direction $x>0$, and the + sign accounts for propagation in the $x<0$ direction. 


\subsection{Travelling waves for Models (1.1) and (1.2)}

After characterizing the spreading properties, the remaining question is that of the existence of invasion profiles. This is what we study here. We consider travelling waves, that is, solutions of the type $u(t, x)=\varphi(x-c t), v(x, y, t)=\psi(x-c t, y)$, where $c \in \mathbb{R}$ is the speed of the wave, and $(u, v)$ approaches the two steady states $(0,0)$ and $(\nu / \mu, 1)$ when $t \rightarrow-\infty$ and $t \rightarrow+\infty$ respectively.

First, consider Model (1.1). We are led to the following elliptic system:

$$
\left\{\begin{aligned}
-c \partial_{x} \psi-d \Delta \psi & =f(\psi), & & x \in \mathbb{R}, y>0 \\
-c \partial_{x} \varphi-D \partial_{x x} \varphi & =\nu \psi-\mu \varphi, & & x \in \mathbb{R}, y=0 \\
-d \partial_{y} \psi & =\mu \varphi-\nu \psi, & & x \in \mathbb{R}, y=0
\end{aligned}\right.
$$

with limiting conditions

$$
\left\{\begin{array}{l}
\varphi(-\infty)=\nu / \mu, \quad \varphi(+\infty)=0, \\
\psi(-\infty, y)=1, \quad \psi(+\infty, y)=0, \text { locally uniformly in } y \geqslant 0
\end{array}\right.
$$

Our main result is the following:

Theorem 2.2. Let $w^{*}>0$ be the asymptotic spreading speed of Theorem 2.1. Then,

(i) For all $c<w^{*}$, there exists no travelling front solution $(\varphi, \psi)$ for $(2.5)-(2.6)$.

(ii) For all $c \geqslant w^{*}$, there exists a travelling front solution $(\varphi, \psi)$ for $(2.5)-(2.6)$. It satisfies $\partial_{x} \varphi<0$ on $\mathbb{R}$ and $\partial_{x} \psi<0$ in $\mathbb{R} \times(0,+\infty)$.

Thus, the classical result for Fisher-KPP extends to our system (1.1): there exist travelling front solutions for a closed half line of speeds bounded from below by the asymptotic speed of spreading for initial data with compact support.

Let us consider next Model (1.2). The travelling wave problem for $u(t, x)=\varphi(x-$ $c t), v(x, y, t)=\psi(x-c t, y)$ is now formulated as follows:

$$
\left\{\begin{aligned}
-c \partial_{x} \psi-d \Delta \psi & =f(\psi), & & x \in \mathbb{R}, y>0 \\
(q-c) \partial_{x} \varphi-D \partial_{x x} \varphi & =\nu \psi-(\mu+\rho) \varphi, & & x \in \mathbb{R}, y=0 \\
-d \partial_{y} \psi & =\mu \varphi-\nu \psi, & & x \in \mathbb{R}, y=0
\end{aligned}\right.
$$

and the limiting conditions are

$$
\begin{cases}\varphi(-\infty)=u_{s}, & \varphi(+\infty)=0, \\ \psi(-\infty, y)=v_{s}(y), & \psi(+\infty, y)=0, \text { locally uniformly in } y \geqslant 0\end{cases}
$$

Here we prove an analogue of Theorem 2.2.

Theorem 2.3. Assume that $f$ satisfies (2.3). Let $w^{*}>0$ be the spreading speed for (1.2). Then,

(i) For all $c<w^{*}$, there exists no travelling front solution $(\varphi, \psi)$ for $(2.7)-(2.8)$.

(ii) For all $c \geqslant w^{*}$, there exists a travelling front solution $(\varphi, \psi)$ for $(2.7)-(2.8)$. It satisfies $\partial_{x} \varphi<0$ on $\mathbb{R}$ and $\partial_{x} \psi<0$ in $\mathbb{R} \times(0,+\infty)$. 


\subsection{The study of Model (1.3)}

Finally, let us consider Model (1.3). Contrary to systems (1.1) and (1.2), nonzero steady states do not exist in some ranges of parameters. Once this issue is settled, we should worry about spreading speeds. Finally, we will introduce the travelling wave problem.

As for Model (1.2), we will need $f$ to satisfy (2.3). In the whole sequel, whenever dealing with Model (1.3), this assumption will be always implicitly understood.

Theorem 2.4. Under the condition

$$
\frac{\mu \sqrt{\rho d}}{\nu+\sqrt{\rho d}} \geqslant f^{\prime}(0)
$$

any solution of (1.3), starting from a bounded initial datum, tends to 0 as $t \rightarrow+\infty$, uniformly in $x \in \mathbb{R}, y \geqslant 0$.

This result means, in particular, that no nonzero steady state exists in the range of parameters (2.9). Conversely, as soon as (2.9) does not hold, the system admits a unique nontrivial steady state, like in Models (1.1) and (1.2).

Theorem 2.5. Under condition

$$
\frac{\mu \sqrt{\rho d}}{\nu+\sqrt{\rho d}}<f^{\prime}(0)
$$

system (1.3) has a unique positive, bounded steady state $\left(u_{s}, v_{s}\right)$. Moreover, $u_{s}$ is constant, equal to the only positive root of

$$
\frac{\mu \sqrt{\rho d}}{\nu+\sqrt{\rho d}} u_{s}=f\left(u_{s}\right)
$$

and $v_{s}=v_{s}(y)=\frac{\mu u_{s}}{\nu+\sqrt{\rho d}} e^{-\sqrt{\rho / d} y}$.

The next question is that of the spreading speed.

Theorem 2.6. Under the condition (2.10), there is a positive spreading speed $w^{*}$. In other words, let $(u, v)$ be a solution of (1.3) with a nonnegative, compactly supported initial datum $\left(u_{0}, v_{0}\right) \not \equiv(0,0)$. Then,

- For all c $>w^{*}$, we have

$$
\lim _{t \rightarrow+\infty} \sup _{|x| \geqslant c t}(u(x, t), v(x, y, t))=(0,0)
$$

- for all $c \in\left[0, w^{*}\right)$, for all $a>0$, we have

$$
\left.\lim _{t \rightarrow+\infty} \sup _{|x| \leqslant c t, 0 \leqslant y \leqslant a} \mid u(x, t), v(x, y, t)\right)-\left(u_{s}, v_{s}(y)\right) \mid=0 .
$$


Theorems 2.4 and 2.6 thus provide a necessary and sufficient condition for extinction or persistence of a species described by model (1.3). In this context, formulas (2.9) and (2.10) lend themselves to an interesting biological interpretation which we now describe.

- First, all else equal, there is a threshold value with respect to the parameter $\mu$ for extinction. That is, there is a value

$$
\mu_{0}:=f^{\prime}(0)\left[\frac{\nu}{\sqrt{\rho d}}+1\right]
$$

such that if $\mu \geqslant \mu_{0}$, there is extinction. In other words there is always a critical rate of loss from the road above which the population goes extinct.

- In particular, if $\mu<f^{\prime}(0)$, then no matter what the other parameters are, there is always persistence. That is, if the loss rate is below the growth rate, there is always persistence.

- Assume now that $\mu>f^{\prime}(0)$. Then, there is an explicit threshold value $\nu_{0}$ for $\nu$ so that there is persistence if and only if $\nu>\nu_{0}$. In other words, a sufficiently large gain term from the field to the road will offset the relatively high loss term.

- Assume again that $\mu>f^{\prime}(0)$ and that $\nu$ is fixed. Then there is a critical threshold $\gamma$ so that there is persistence of the species if and only if $\rho d<\gamma$. Thus, all else equal, too large a value of $d$, the diffusion in the field, is lethal for the species. Likewise, too large a death rate in the field will offset the influence of the road and becomes lethal. It is worthy to note that only the product $\rho d$ comes into play. Thus, for instance, a large mortality rate in the field may be offset by a slow diffusion.

We can now state an existence result of travelling waves that is a counterpart of Theorem 2.6. The problem for $u(t, x)=\varphi(x-c t), v(x, y, t)=\psi(x-c t, y)$ reads as follows.

$$
\left\{\begin{aligned}
-c \partial_{x} \psi-d \Delta \psi & =-\rho \psi, & & x \in \mathbb{R}, y>0 \\
-c \partial_{x} \varphi-D \partial_{x x} \varphi+\mu \varphi-\nu \psi & =f(\varphi), & & x \in \mathbb{R}, y=0 \\
-d \partial_{y} \psi & =\mu \varphi-\nu \psi, & & x \in \mathbb{R}, y=0
\end{aligned}\right.
$$

and the limiting conditions are

$$
\begin{cases}\varphi(-\infty)=u_{s}, & \varphi(+\infty)=0 \\ \psi(-\infty, y)=v_{s}(y), & \psi(+\infty, y)=0, \text { locally uniformly in } y \geqslant 0\end{cases}
$$

The existence result is the following

Theorem 2.7. Under the condition (2.10), let $w^{*}$ be the spreading speed given by Theorem 2.6. For all $c \geqslant w^{*}$, there is an $x$-decreasing solution to (2.11)-(2.12), and no solution for $c<w^{*}$. 
The paper is organized as follows. In the next section we first show that no front can travel with speed less than $w^{*}$ in the $x$-direction, that is, Theorem 2.2 (i). The same proof will be valid for Theorems 2.3 and 2.7, (i). We then turn, in Section 4, to Model (1.3), and prove the general extinction result in the absence of steady solutions, that is, Theorem 2.4. Section 5 is devoted to the proof of Theorem 2.5. In Section 6 we recall the construction of the asymptotic speed of propagation $w^{*}$ in Models (1.1) and (1.2), then we carry the computations for (1.3) and prove Theorem 2.6. In Section 7, we prove the existence of travelling waves for Model (1.1). We will see that an important ingredient is the existence of planar waves, which act a super solutions. Lastly, in Section 8 we discuss the adaptation of the method to Models (1.2) and (1.3).

\section{Lower bound on the speed of travelling fronts}

We consider here system (1.1). In a moving frame with speed $c \in \mathbb{R}$ in the $x$ direction this system reads

$$
\left\{\begin{aligned}
\partial_{t} v-c \partial_{x} v-d \Delta v & =f(v), & & x \in \mathbb{R}, y>0, t>0, \\
\partial_{t} u-c \partial_{x} u-D \partial_{x x} u & =\nu v-\mu u, & & x \in \mathbb{R}, y=0, t>0, \\
-d \partial_{y} v & =\mu u-\nu v, & & x \in \mathbb{R}, y=0, \quad t>0,
\end{aligned}\right.
$$

The well-posedness for the associated Cauchy problem with continuous initial datum is derived in [9] (see also [10]). The proof works exactly in the same way for System (1.3). The solutions we deal with in this paper are always nonnegative and bounded. Let us quote from [9] the strong comparison principle.

Proposition $3.1([9])$. Let $(\underline{u}, \underline{v})$ and $(\bar{u}, \bar{v})$ be respectively a subsolution bounded from above and a supersolution bounded from below of (3.1) satisfying $\underline{u} \leqslant \bar{u}$ and $\underline{v} \leqslant \bar{v}$ at $t=0$. Then, either $\underline{u}<\bar{u}$ and $\underline{v}<\bar{v}$ for all $t$, or there exists $T>0$ such that $(\underline{u}, \underline{v})=(\bar{u}, \bar{v})$ for $t \leqslant T$.

We show now how the non-existence of fronts with slow speed is immediately obtained from the spreading result of [9].

Proof of Theorem 2.2, part (i). Observe preliminarily that, owing to Proposition 3.1, we do not need the requirement that the initial datum $\left(u_{0}, v_{0}\right)$ has compact support in the second statement of Theorem 2.1 (i). Indeed, one can always consider a solution with a compactly supported initial datum lying below $\left(u_{0}, v_{0}\right)$ to which apply the result, and then obtain the conclusion for $(u, v)$ by comparison.

Suppose that problem (2.5)-(2.6) admits a solution $(\varphi, \psi)$, for some $c \in \mathbb{R}$. Then $(\varphi(x-c t), \psi(x-c t, y))$ is a solution of system (1.1). We can apply the second statement of Theorem 2.1 (i) and deduce

$$
\forall c^{\prime} \in\left[0, w^{*}\right), \quad \forall a>0, \quad \lim _{t \rightarrow+\infty} \sup _{|x| \leqslant c^{\prime} t, 0 \leqslant y \leqslant a}|(\varphi(x-c t), \psi(x-c t, y))-(\nu / \mu, 1)|=0 .
$$

In particular, for any $c^{\prime} \in\left[0, w^{*}\right)$, we find that

$$
\lim _{t \rightarrow+\infty} \varphi\left(\left(c^{\prime}-c\right) t\right)=\nu / \mu,
$$


and therefore (2.6) implies that $c^{\prime} \leqslant c$. Since this is true for any $c^{\prime} \in\left[0, w^{*}\right)$, we eventually obtain $c \geqslant w^{*}$.

The proof of that fact for Systems (1.2) and (1.3) (when $w^{*}>0$ ) is the same.

\section{Condition for extinction in system (1.3)}

We now show that extinction always occurs for Model (1.3) when the reaction $f$ is not strong enough, in the sense that (2.9) holds. Namely, we prove Theorem 2.4. This is done by deriving two simple properties concerning, respectively, the large time behaviour of general solutions and the existence of nontrivial, $x$-independent steady states.

Lemma 4.1. Any solution $(u, v)$ to (1.3) satisfies

$$
\limsup _{t \rightarrow+\infty} u(t, x) \leqslant \widehat{u}, \quad \limsup _{t \rightarrow+\infty} v(t, x, y) \leqslant \widehat{v}(y)
$$

uniformly in $x \in \mathbb{R}$ and $y \geqslant 0$, where $(\widehat{u}, \widehat{v}(y))$ is a nonnegative, bounded, $x$-independent steady state.

Proof. Direct computation shows that, for $K \geqslant 1$ large enough, the pair $K(1, \mu / \nu)$ is a supersolution to (1.3) and it is larger than the initial datum of $(u, v)$. By the comparison principle - which is the analogous of Proposition 3.1 but for system (1.3) the solution $(\bar{u}, \bar{v})$ with initial datum $K(1, \mu / \nu)$ remains above $(u, v)$ for all positive times. Moreover, always because of the comparison principle, $(\bar{u}, \bar{v})$ is $x$-independent and nonincreasing in $t$. It follows that, as $t \rightarrow+\infty$, it converges locally uniformly in $y \geqslant 0$ to an $x$-independent steady state $(\widehat{u}, \widehat{v}(y))$. It remains to prove that the convergence is uniform in $y \geqslant 0$. For $\varepsilon>0$, let $T_{\varepsilon}>0$ be such that $\bar{v}(t, 0) \leqslant \widehat{v}(0)+\varepsilon$ for $t \geqslant T_{\varepsilon}$. Consider the function

$$
v_{\varepsilon}(t, y):=\widehat{v}(y)+\varepsilon+K \frac{\mu}{\nu} e^{-\rho\left(t-T_{\varepsilon}\right)} .
$$

This is a supersolution of the first equation of (1.3). Furthermore, it satisfies

$$
v_{\varepsilon}\left(T_{\varepsilon}, y\right)>K \frac{\mu}{\nu}=\max \bar{v}, \quad v_{\varepsilon}(t, 0) \geqslant \widehat{v}(0)+\varepsilon \geqslant \bar{v}(t, 0) \quad \text { for all } t \geqslant T_{\varepsilon} .
$$

Hence, applying the comparison principle, we derive $\bar{v}(t, y) \leqslant v_{\varepsilon}(t, y)$ for $t>T_{\varepsilon}$ and $y \geqslant 0$, and therefore $\bar{v}(t, y) \leqslant \widehat{v}(y)+2 \varepsilon$ for $t$ large enough and $y \geqslant 0$. This concludes the proof of the lemma.

Let us mention that it could happen that $(\widehat{u}, \widehat{v}(y))$ is the trivial solution $(0,0)$. Actually, this is what always happens when (2.10) does not hold.

Lemma 4.2. Model (1.3) has a (unique) positive, $x$-independent steady state $\left(u_{s}, v_{s}(y)\right)$ if and only if (2.10) holds. 
Proof. Let $(u, v(y))$ be an $x$-independent steady state. The equation in the field and the exchange condition yield, respectively,

$$
v(y)=v(0) e^{-\sqrt{\rho / d} y}, \quad v(0)=\frac{\mu u}{\nu+\sqrt{\rho d}} .
$$

Inserting this in the equation for $u$ yields

$$
\frac{\mu \sqrt{\rho d}}{\nu+\sqrt{\rho d}} u=f(u)
$$

Since, by Assumption $(\mathrm{H})$, the function $(0,1] \ni u \mapsto f(u) / u$ decreases from $f^{\prime}(0)$ to 0 , the above equation has a unique solution $u_{s} \in(0,1)$ if and only if $(2.10)$ holds.

We point out that Lemma 4.2 is much weaker than Theorem 2.5, the latter excluding the existence of $x$-dependent steady states. As we will see in the next section, some additional work is needed to get rid of the $x$-dependence.

Proof of Theorem 2.4. Let $(u, v)$ be a solution to System (1.3). Because (2.10) is not satisfied, Lemma 4.2 implies that the steady state given by Lemma 4.1 is $(\widehat{u}, \widehat{v}(y)) \equiv$ $(0,0)$. Hence, $(u, v) \rightarrow(0,0)$ uniformly in space as $t \rightarrow+\infty$.

We conclude this section by showing that, when condition (2.10) is violated with a strict inequality, extinction occurs exponentially fast in time.

Proposition 4.3. Assume that $f^{\prime}(0)<\frac{\mu \sqrt{\rho d}}{\nu+\sqrt{\rho d}}$. Then there exists $\delta>0$ such that

$$
0 \leqslant u(t), v(t, y) \leqslant C e^{-\delta t}
$$

Proof. Because $f(s) \leqslant f^{\prime}(0) s$, it is sufficient to argue on the linear system

$$
\left\{\begin{aligned}
\dot{u}+\mu u-\nu v(t, 0) & =f^{\prime}(0) u & & t>0 \\
-d v_{y}+\nu v & =\mu u(t) & & t>0, y=0 \\
v_{t}-d v_{y y} & =-\rho v & & t>0, y>0
\end{aligned}\right.
$$

with initial datum $\left(u_{0}, v_{0}\right)$. One could prove (4.1) via a Laplace transform argument, but that would be a little tedious. We prefer to argue via a supersolution argument, that will be less precise, but shorter and more transparent. In order to get rid of the initial datum $v_{0}$, we define $\bar{v}(t):=\left\|v_{0}\right\|_{\infty} e^{-\rho t}$ and we introduce the new unknown $w(t, y)=v(t, y)-\bar{v}(t)$. The system for $(u, w)$ reads

$$
\left\{\begin{aligned}
\left(\partial_{t}-d \partial_{y y}+\rho\right) w & =0 & & t>0, y>0 \\
-d w_{y}+\nu(w+\bar{v}) & =\mu u & & t>0, y=0 \\
\dot{u}+\mu u-\nu(w+\bar{v}) & =f^{\prime}(0) u & & t>0, y=0
\end{aligned}\right.
$$

with $w(0, y) \leqslant 0$. The game is to find a super-solution $(\bar{u}(t), \bar{w}(t, y))$ to the new system. We first choose

$$
\bar{w}(t, y)=M e^{-y \sqrt{(\rho-\delta) / d}-\delta t}
$$


with $M>0$ large and $\delta>0$ small to be chosen later. The function $\bar{w}$ solves the equation for $w$ in the field. Then we decide to satisfy the exchange condition exactly, namely we choose

$$
\mu \bar{u}(t)=(\sqrt{d(\rho-\delta)}+\nu) M e^{-\delta t}+\nu\left\|v_{0}\right\|_{\infty} e^{-\rho t} .
$$

We now only have to choose $M$ and $\delta$ so that $(\bar{u}, \bar{w})$ satisfies the third equation of (4.3), maybe not with an $=$ sign, but at least with $a \geqslant$ sign. By computation, there exists $C>0$ independent of $\delta, M$ such that

$$
\begin{aligned}
\dot{\bar{u}}+\mu \bar{u}-\nu(\bar{w}+\bar{v})-f^{\prime}(0) \bar{u} \geqslant & -C \delta M e^{-\delta t}-\frac{\rho \nu}{\mu}\left\|v_{0}\right\|_{\infty} e^{-\rho t}+\sqrt{d(\rho-\delta)} M e^{-\delta t}-f^{\prime}(0) \bar{u} \\
\geqslant & \left(-C \delta+\sqrt{d(\rho-\delta)}-f^{\prime}(0) \frac{\sqrt{d(\rho-\delta)}+\nu}{\mu}\right) M e^{-\delta t} \\
& -\frac{\nu}{\mu}\left(\rho+f^{\prime}(0)\right)\left\|v_{0}\right\|_{\infty} e^{-\rho t} .
\end{aligned}
$$

By hypothesis, the factor in front of $M e^{-\delta t}$ of the RHS is positive for $\delta=0$. We can therefore choose $\delta>0$ sufficiently small in such a way that this remains true. Then, taking in addition $\delta<\rho$, we find that the RHS is positive for $M$ large enough. At the expense of increasing $M$ a little more, we also obtain $(u(0), w(0, y)) \leqslant(\bar{u}(0), \bar{w}(0, y))$. This implies

$$
(u(t), w(t, y)) \leqslant(\bar{u}(t), \bar{w}(t, y)),
$$

which concludes the proof.

\section{$5 \quad$ The steady states of Model (1.3)}

Let us assume (2.10) to hold. We know from Lemma 4.2 that in this case the system admits a unique one-dimensional equilibrium $\left(u_{s}, v_{s}(y)\right)$. Our task is now to prove that there are no $x$-dependent equilibria, and we are going to do it in the

Proof of Theorem 2.5. What is behind this result is nothing else than the instability of the steady state $(0,0)$, and $(2.10)$ can be seen as a necessary and sufficient condition for that. Let us confirm it by performing a linear stability analysis of the $(0,0)$ state. We first consider the $x$-independent, one-dimensional linear system

$$
\left\{\begin{array}{l}
-d v^{\prime \prime}(y)+\rho v(y)=\lambda v(y), \quad y>0 \\
\mu u-\nu v(0)=f^{\prime}(0) u+\lambda u \\
-d v^{\prime}(0)=\mu u-\nu v(0)
\end{array}\right.
$$

A solution $\lambda<0, u>0, v(y)>0$ bounded, exists if and only if

$$
\frac{\mu \sqrt{(\rho-\lambda) d}}{\nu+\sqrt{(\rho-\lambda) d}}=f^{\prime}(0)+\lambda .
$$

The LHS of (5.1) decreases from $\mu$ to $\frac{\mu \sqrt{\rho d}}{\nu+\sqrt{\rho d}}$ as $\lambda$ increases from $-\infty$ to 0 , whereas the RHS increases from $-\infty$ to $f^{\prime}(0)$. From the condition (2.10), equation (5.1) has a 
unique solution $\lambda_{0}<0$, and one may choose a positive corresponding eigenfunction, as can easily seen by inspection. In order to work with these, we need to check that the $(0,0)$ state is also unstable for the Dirichlet problem in some sufficiently large domain; in [9] it was sufficient to perform the analysis in large balls far from the road, but this is not possible here because, in order to take advantage of the reaction term $f$, we need to consider a domain touching the road. Take $L>0$ and consider the problem

$$
\begin{cases}-d \Delta v+\rho v=\lambda v, & x \in(-L, L), y>0 \\ v(x, L)=0, & x \in(-L, L) \\ -D \partial_{x x} u+\mu u-\nu v=f^{\prime}(0) u+\lambda u, & x \in(-L, L), y=0 \\ -d \partial_{y} v=\mu u-\nu v, & x \in(-L, L), y=0 \\ u( \pm L)=v( \pm L, 0)=0 . & \end{cases}
$$

We may look for a solution of the form

$$
u_{L}(x)=\widetilde{u} \cos \left(\frac{\pi}{2 L} x\right), \quad v_{L}(x, y)=\widetilde{v}(y) \cos \left(\frac{\pi}{2 L} x\right) .
$$

with $\widetilde{u}, \widetilde{v}>0$. The first two equations of (5.2) yield

$$
\widetilde{v}(y)=\frac{\widetilde{v}(0)}{1-e^{-2 \omega_{L} L}}\left(e^{-\omega_{L} y}-e^{\omega_{L}(y-2 L)}\right), \quad \text { where } \omega_{L}:=\sqrt{\frac{\rho-\lambda}{d}+\frac{\pi^{2}}{4 L^{2}}} .
$$

Then, by explicit computation, the eigenvalue problem (5.2) reduces to the algebraic equation

$$
\frac{\mu d_{L} \omega_{L}}{\nu+d_{L} \omega_{L}}=f^{\prime}(0)+\lambda-\frac{D \pi^{2}}{4 L^{2}}, \quad \text { where } \quad d_{L}:=d \frac{1+e^{-2 \omega_{L} L}}{1-e^{-2 \omega_{L} L}}
$$

For $L \rightarrow+\infty$, (5.3) is a perturbation of (5.1), and so has a unique root $\lambda_{L}=\lambda_{0}+$ $O\left(L^{-2}\right)<0$. From now on, choose $L_{0}>0$ large enough so that the above is true for $L=L_{0}$. Denote by $\left(u_{L_{0}}(x), v_{L_{0}}(x, y)\right)$ the corresponding eigenfunction. A well-known consequence of the fact that $\lambda_{L_{0}}<0$ - the computation can easily be done - is that, for $\varepsilon>0$ small enough, the couple $\varepsilon\left(u_{L_{0}}(x), v_{L_{0}}(x, y)\right)$ is a subsolution to (1.3) in the rectangle $[-L, L] \times[0, L]$, and thus, extending it by 0 outside the rectangle, it is a generalized subsolution (in the sense of [9, Proposition 3.3]).

We now follow the lines of [9], based on a variant of the sliding method of [7]. We will actually show that any nontrivial solution $(u, v)$ of $(1.3)$ converges, as $t \rightarrow+\infty$, to the unique $x$-independent steady state $\left(u_{s}, v_{s}\right)$ locally uniformly in space; in particular, there are no other steady states.

So, let $(u, v) \not \equiv(0,0)$ be a solution of the Cauchy problem. Lemma 4.1 provides us with the upper bound $(\widehat{u}, \widehat{v}(y))$ for the large-time behaviour of $(u, v)$. Let us derive now a lower bound, which will also show that $(\widehat{u}, \widehat{v}(y)) \not \equiv(0,0)$ and thus $(\widehat{u}, \widehat{v}(y)) \equiv$ $\left(u_{s}, v_{s}(y)\right)$. By the strong maximum principle, $u$ and $v$ are strictly positive at, say, $t=$ 1. Take $\varepsilon>0$ small enough in such a way that the generalized subsolution $\varepsilon\left(u_{L_{0}}, v_{L_{0}}\right)$ lies below the pair $(u, v)$ at $t=1$. The solution $(\underline{u}, \underline{v})$ starting from $\varepsilon\left(u_{L_{0}}, v_{L_{0}}\right)$ at $t=1$ stays below $(u, v)$ for all $t>1$ and, moreover, it increasing in $t$. Therefore, as $t \rightarrow+\infty$, it converges locally uniformly in space to a steady state $(\check{u}, \check{v})$ lying 
between $\varepsilon\left(u_{L_{0}}, v_{L_{0}}\right)$ and $(\widehat{u}, \widehat{v})$. Note that, owing to the strong maximum principle, $\check{u}(x)>\varepsilon u_{L_{0}}(x)$ and $\check{v}(x, y)>\varepsilon v_{L_{0}}(x, y)$ and therefore, because $\varepsilon\left(u_{L_{0}}, v_{L_{0}}\right)$ has compact support, the inequalities remain true even if we slide the subsolution a bit, namely,

$$
\check{u}(x)>\varepsilon u_{L_{0}}(x+\tau), \quad \check{v}(x, y)>\varepsilon v_{L_{0}}(x+\tau, y),
$$

provided $|\tau|$ is small enough. Comparing then the stationary solution $(\check{u}, \breve{v})$ with the solution emerging from $\varepsilon\left(u_{L_{0}}(x+\tau), v_{L_{0}}(x+\tau, y)\right)$, which is nothing else that $(\underline{u}, \underline{v})$ translated in $x$ by $\tau$, and letting $t \rightarrow+\infty$, we eventually infer that $(\check{u}, \check{v})$ is larger than $(\check{u}, \check{v})$ itself translated in $x$ by $\tau$. This means that $(\check{u}, \check{v})$ is $x$-independent. Since we know from Lemma 4.2 that there is a unique $x$-independent, positive, stationary solution $\left(u_{s}, v_{s}\right)$, we conclude that $(\check{u}, \check{u}) \equiv(\widehat{u}, \widehat{v}) \equiv\left(u_{s}, v_{s}\right)$, and therefore $(u, v) \rightarrow$ $\left(u_{s}, v_{s}\right)$ as $t \rightarrow+\infty$, locally uniformly in space.

\section{Construction of the asymptotic speed of spreading}

We recall here from [9] the construction of the spreading speed $w^{*}$. We obtained $w^{*}$ from the system giving exponential solutions for the linearised problem

$$
\begin{cases}\partial_{t} v-d \Delta v=f^{\prime}(0) v, & x \in \mathbb{R}, y>0, t \in \mathbb{R}, \\ \partial_{t} u-D \partial_{x x} u=\nu v-\mu u, & x \in \mathbb{R}, y=0, t \in \mathbb{R}, \\ -d \partial_{y} v=\mu u-\nu v, & x \in \mathbb{R}, y=0, t \in \mathbb{R} .\end{cases}
$$

Looking for solutions of (6.1) of the form

$$
(u(x, t), v(x, y, t))=\left(e^{-\alpha(x-c t)}, \gamma e^{-\alpha(x-c t)-\beta y}\right),
$$

with $\alpha, \gamma>0$ and $\beta \in \mathbb{R}$ (not necessarily positive) leads to the following system:

$$
\left\{\begin{aligned}
-d\left(\alpha^{2}+\beta^{2}\right)+c \alpha & =f^{\prime}(0) \\
-D \alpha^{2}+c \alpha & =\nu \gamma-\mu \\
d \beta \gamma & =\mu-\nu \gamma
\end{aligned}\right.
$$

With respect to the stationary travelling front system, we observe that when $\alpha, \beta, \gamma$ solve (6.3), then

$$
(\bar{\varphi}(x), \bar{\psi}(x, y))=\left(e^{-\alpha x}, \gamma e^{-\alpha x-\beta y}\right)
$$

is a supersolution of $(2.5)$ due to the KPP defining property $f(s) \leqslant f^{\prime}(0) s$, and also of the limiting condition (2.6).

We recall the analysis of [9]. In the $(\beta, \alpha)$ plane, the first equation of $(6.3)$ is that of the circle $\Gamma_{c, d}$ with centre $\left(0, \frac{c}{2 d}\right)$ and radius $\frac{\sqrt{c^{2}-c_{K}^{2}}}{2 d}$. The second equation represents a curve $\Gamma_{c, D}$ passing through the points $(0,0)$ and $\left(0, \frac{c}{D}\right)$, made up of two branches:

$$
\alpha=\alpha_{D}^{ \pm}(c, \beta)
$$


$\alpha_{D}^{+}$increasing in $\beta, \alpha_{D}^{-}$decreasing in $\beta$, joining at the point $\left(\beta_{\text {lim }}, \alpha_{\text {lim }}\right)$ with

$$
\beta_{l i m}=\frac{1}{d}\left(\frac{\mu}{c^{2} / 4 D+\mu}-\nu\right), \alpha_{l i m}=\frac{c}{2 D} .
$$

This point is the leftmost point of $\Gamma_{c, D}$. The construction of the spreading speed is from then on quite intuitive: first, if $D>2 d$, then $\Gamma_{c, D}$ is outside $\Gamma_{c, d}$; for very large $c$ one my check that the interior of $\Gamma_{c, D}$ contains points of the interior of $\Gamma_{c, d}$. This implies, increasing $c$ from $c_{K}$ to infinity, the existence of $c^{*}$ such that $\Gamma_{c, D}$ and $\Gamma_{c, d}$ are tangent at one point. This minimum value is $w^{*}$. Second, if $D \leqslant 2 d, \Gamma_{c, d}$ is inside the interior of $\Gamma_{c, D}$. So, for all $c \geqslant c_{K}$, there is a linear supersolution to (6.1). Set $c_{K}:=w^{*}$. To prove that $w^{*}$ is the spreading speed, the main step is to prove lower estimates, that are obtained by approximating (1.1) in infinite strips of larger and larger widths, see [9]. The same considerations hold for System (1.2), see [10]. So, let us turn to model (1.3).

Proof of Theorem 2.6. The first task is to look for exponential solutions for

$$
\begin{cases}\partial_{t} v-d \Delta v=-\rho v, & x \in \mathbb{R}, y>0, t \in \mathbb{R} \\ \partial_{t} u-D \partial_{x x} u=\nu v-\mu u+f^{\prime}(0) u, & x \in \mathbb{R}, y=0, t \in \mathbb{R} \\ -d \partial_{y} v=\mu u-\nu v, & x \in \mathbb{R}, y=0, t \in \mathbb{R}\end{cases}
$$

Once this is done, the proof goes exactly as in [9].

Looking for solutions of the form (6.2) yields

$$
\left\{\begin{aligned}
-d\left(\alpha^{2}+\beta^{2}\right)+c \alpha & =-\rho \\
-D \alpha^{2}+c \alpha+\frac{d \beta \mu}{\nu+d \beta} & =f^{\prime}(0)
\end{aligned}\right.
$$

In the $(\beta, \alpha)$ plane, the first equation of $(6.4)$ is that of the circle $\Gamma_{c, d}$ with centre $\left(0, \frac{c}{2 d}\right)$ and radius

$$
\frac{\sqrt{c^{2}+4 d \rho}}{2 d}>\frac{c}{2 d}
$$

As for the second equation, it has solutions if and only if

$$
c^{2}+4 D\left(\mu-f^{\prime}(0)-\frac{\mu \nu}{\nu+d \beta}\right) \geqslant 0 .
$$

We are going to study two different cases. They will in the end produce results of the same kind, but we believe that it is of interest to distinguish them, because they deal with a biologically interesting threshold.

Case 1. $\mu>f^{\prime}(0)$. This condition means that the road yields to the field more than what it produces. Then, for all $c \geqslant 0$, the second equation of (6.4) represents two branches $\alpha=\alpha_{D}^{ \pm}(c, \beta)$ whose union we call $\Gamma_{c, D}$ and which meet at the point $\left(\beta_{\text {lim }}(c), \alpha_{\text {lim }}(c)\right)$, with

$$
d \beta_{\text {lim }}(c)=\frac{\nu\left(f^{\prime}(0)-c^{2} / 4 D\right)}{\mu-f^{\prime}(0)+c^{2} / 4 D}, \quad \alpha_{l i m}(c)=\frac{c}{2 D} .
$$


This point is the leftmost point of $\Gamma_{c, D}$. Inspection of the value of $\alpha_{D}^{ \pm}$reveals that $\alpha_{D}^{-}(c, \beta)<0$ for large $\beta$. It will be useful to have in mind the following values:

$$
d \beta_{\text {lim }}(0)=\frac{\nu f^{\prime}(0)}{\mu-f^{\prime}(0)}, \quad \alpha_{l i m}(c)=0 .
$$

Case 2. $\mu \leqslant f^{\prime}(0)$. This means that the reproduction rate when the density of the species is very small is larger than what is yielded to the road. Condition (6.6) for the existence of solutions means that (6.4) has a solution only if $c>4 D\left(f^{\prime}(0)-\mu\right)$, a condition that is almost void if $f^{\prime}(0)=\mu$. For $c>4 D\left(f^{\prime}(0)-\mu\right)$, the properties of these solutions are the same as in the preceding case.

Let us study how $\Gamma_{c, d}$ and $\Gamma_{c, D}$ intersect. For this we go by the above two cases. In the case $\mu>f^{\prime}(0)$, let us first investigate whether or not $c=0$ is an acceptable speed. This is equivalent to saying that the radius of $\Gamma_{0, d}$ is less than $\beta_{l i m}(0)$, in other words, using (6.8): $f^{\prime}(0) \leqslant \frac{\mu \sqrt{\rho d}}{\mu-f^{\prime}(0)}$, a contradiction with (2.10). So, $\Gamma_{0, D}$ is strictly to the right of $\Gamma_{0, d}$ and we need to increase $c$ in order to get a tangency point. Because of (6.7), we have on the one hand

$$
\lim _{c \rightarrow+\infty} \beta_{\text {lim }}(c)=-\frac{\nu}{d}
$$

On the other hand, because of (6.5), $\Gamma_{c, d} \cap\{\alpha<0\}$ is non void, for all c. So, $\Gamma_{c, d}$ and $\Gamma_{c, D}$ have to cross for some $c$, and, for that, must have a common tangency point. This defines $w^{*}$, and it is a positive number.

In the case $\mu \leqslant f^{\prime}(0)$, we have, unconditionally, $w^{*}>0$. Indeed, for $c$ slightly larger than $\sqrt{\frac{c^{2}}{4 D}-\left(f^{\prime}(0)-\mu\right)}$, then $\left(\beta_{\text {lim }}(c), \alpha_{\text {lim }}(c)\right)$ is very much to the right of $\Gamma_{c, d}$. From the same considerations as before, there is $w^{*}>0$ for which $\Gamma_{c, D}$ and $\Gamma_{c, d}$ are tangent.

Now, the rest of the proof follows the same lines as in [9]. On the one hand, one sees that the spreading speed cannot be larger than $w^{*}$ by comparison with the exponential (super)solution moving with speed $c=w^{*}$. For the lower bound, one constructs compactly supported generalized subsolutions for $c<w^{*}$ close to $w^{*}$ by using Rouché's theorem. These are the key ingredient to derive the large-time convergence to $\left(u_{s}, v_{s}\right)$ for solutions of the problem in the moving frame with speed $c$ in the $x$-direction, which is precisely what one needs to prove. Namely, one repeats the argument of the proof of Theorem 2.5 above, using these generalized subsolutions in place of the pair $\varepsilon\left(u_{L_{0}}(x), v_{L_{0}}(x, y)\right)$.

\section{$7 \quad$ Existence of travelling fronts in System (1.1)}

In this section, we prove part (ii) of Theorem 2.2, that is, the existence of travelling front solutions of $(2.5)-(2.6)$ when $c \geqslant w^{*}$, as well as their monotonicity in $x$.

Throughout this section, $c \geqslant w^{*}$ is fixed. To construct a solution of (2.5)-(2.6), we are going to approximate the half-plane $\mathbb{R} \times(0,+\infty)$ by the finite truncations

$$
\Omega_{a b}:=(-a, a) \times(0, b),
$$


with $a, b$ positive parameters. The construction follows some ideas of [6] and [3] which modify the approach in [8], based on the sliding method. To start the method one needs to have a supersolution connecting the steady states $(\nu / \mu, 1)$ and $(0,0)$ which is strictly decreasing in $x$. The natural candidate would be the exponential pair $(\bar{\varphi}, \bar{\psi})$ defined in the previous section. In order to fulfil the limiting condition, this needs to be truncated by $(\nu / \mu, 1)$, but doing so it is no longer strictly monotone in $x$. An extra argument is therefore required.

Lemma 7.1. There exists a pair $(\Phi, \Psi)$ which is a supersolution of the first two equations of (2.5) and a solution of the third one. Moreover, $\Phi$ and $\Psi$ are strictly decreasing in $x$ and satisfy (2.6).

Proof. Because $c \geqslant w^{*}$, system (6.3) admits a solution $\alpha, \beta, \gamma$ with $\alpha, \gamma>0$. That is,

$$
(\bar{\varphi}(x), \bar{\psi}(x, y))=\left(e^{-\alpha x}, \gamma e^{-\alpha x-\beta y}\right)
$$

is a supersolution of the travelling front problem (2.5). Let $(u, v)$ be the solution of the system in the moving frame (3.1) with initial datum

$$
(\min \{\bar{\varphi}(x), \nu / \mu\}, \min \{\bar{\psi}(x, y), 1\}) .
$$

Since both $(\bar{\varphi}, \bar{\psi})$ and $(\nu / \mu, 1)$ are supersolutions of $(3.1)$, it follows from the comparison principle - Proposition 3.1 - that $(u, v)$ is nonincreasing in $t$ and that it is nonincreasing in $x$ because the initial datum is. Suppose that the monotonicity in $x$ is not strict for some $T>0$, that is, there exists $h>0$ such that

$$
\min _{x \in \mathbb{R}}(u(x, T)-u(x+h, T))=0 \quad \text { or } \quad \min _{x \in \mathbb{R}, y \geqslant 0}(v(x, y, T)-v(x+h, y, T))=0 .
$$

The strong comparison principle implies that $(u, v)$ coincides with its $x$-translation by $h$ for all $t \leqslant T$, which is not true at $t=0$. This shows that $(u, v)$ is strictly decreasing in $x$ for all $t>0$. Observe that, because $\partial_{t} u, \partial_{t} v \leqslant 0,(u, v)$ is a supersolution of the stationary problem (2.5) for any $t>0$ frozen. We then define

$$
\Phi(x):=u(x, 1), \quad \Psi(x, y):=v(x, y, 1) .
$$

It only remains to check that $(\Phi, \Psi)$ satisfies the limiting conditions (2.6). For the limit at $+\infty$, we already know that

$$
\Phi(+\infty)=u(+\infty, 1) \leqslant \bar{\varphi}(+\infty)=0, \quad \Psi(+\infty, y)=v(+\infty, y, 1) \leqslant \bar{\psi}(+\infty, y)=0 .
$$

Consider now a sequence $\left(x_{n}\right)_{n \in \mathbb{N}}$ diverging to $-\infty$. Then, by standard parabolic estimates (see, e.g., [20]) the sequence of translations

$$
\left(u\left(x+x_{n}, t\right), v\left(x+x_{n}, y, t\right)\right)
$$

converges locally uniformly in $x \in \mathbb{R}, y \geqslant 0, t \geqslant 0$, to the solution of (3.1) with initial datum $(\nu / \mu, 1)$, that is, to the constant function $(\nu / \mu, 1)$. In particular, $\Phi(-\infty)=\nu / \mu$, $\Psi(-\infty, y)=1$. 
Notice that, since the problem (2.5) is invariant under $x$-translations, the pair

$$
\left(\Phi_{h}(x), \Psi_{h}(x, y)\right):=(\Phi(x+h), \Psi(x+h, y))
$$

is still a supersolution of system (2.5), for any $h \in \mathbb{R}$.

Another technical difficulty in the application of the sliding method is that we need to impose a boundary datum on the right face which coincides with a subsolution of the system. This will be achieved by setting

$$
k_{a}^{b}:=\min \left\{\frac{\mu}{\nu} \Phi(a), \min _{y \in[0, b]} \Psi(a, y)\right\} .
$$

We can now consider the problem in the truncated domain $\Omega_{a b}$ for unknowns $w=w(x, y)$ and $z=z(x)$ meant to approximate $\psi$ and $\varphi$ respectively:

$$
\begin{cases}-c \partial_{x} w-d \Delta w=f(w), & (x, y) \in \Omega_{a b}=(-a, a) \times(0, b), \\ -c \partial_{x} z-D \partial_{x x} z=\nu w-\mu z, & x \in(-a, a), y=0, \\ -d \partial_{y} w=\mu z-\nu w, & x \in(-a, a), y=0, \\ w(x, y)=\frac{a-x}{2 a} \Psi_{h}(x, y)+\frac{a+x}{2 a} k_{a+h}^{b}, & (x, y) \in \partial \Omega_{a b}, y \neq 0, \\ z(-a)=\Phi_{h}(-a), \quad z(a)=\frac{\nu}{\mu} k_{a+h}^{b} . & \end{cases}
$$

For fixed parameters $a, b>0$, we require the following.

Proposition 7.1. For all $h \in \mathbb{R}$, there exits a unique solution $(z, w)$ of $(7.3)$ satisfying

$$
\frac{\nu}{\mu} k_{a+h}^{b} \leqslant z \leqslant \Phi_{h}, \quad k_{a+h}^{b} \leqslant w \leqslant \Psi_{h}
$$

Moreover, this solution is nonincreasing in $x$ and it is continuous with respect to the parameters $c, h$.

Proof. The proof is divided into three parts. In the first two, we assume without loss of generality that $h=0$.

Existence and lower bound.

Explicit computation shows that $k_{a}^{b}(\nu / \mu, 1)$ and $(\Phi, \Psi)$ are respectively a subsolution and a supersolution of (7.3). By Proposition A.1 in the Appendix - notice that the datum satisfies the compatibility condition (A.2) - there exists a solution of problem (7.3) satisfying (7.4). Let us derive the qualitative properties of this solution. First, we know from (7.4) that the function $\widehat{z}:=z-\frac{\nu}{\mu} k_{a}^{b}$ is nonnegative in $(-a, a)$ and that there satisfies

$$
-c \partial_{x} \widehat{z}-D \partial_{x x} \widehat{z}+\mu \widehat{z}=\nu w(x, 0)-\nu k_{a}^{b} \geqslant 0 .
$$

Since $\widehat{z}(-a)>0$, it follows from the strong maximum principle (see for instance [22]) that $\widehat{z}>0$ in $(-a, a)$. Assume now by contradiction that there exists $\left(x_{0}, y_{0}\right) \in$ $[-a, a) \times[0, b]$ such that $w\left(x_{0}, y_{0}\right)=k_{a}^{b}$. We know that $x_{0} \neq-a$ because $w(-a, y)=$ 
$\Phi(-a, y)>k_{a}^{b}$. Moreover, $y_{0} \notin(0, b)$ because otherwise using $f\left(k_{a}^{b}\right)>0$ and the strong maximum principle one would get $w \equiv k_{a}^{b}$. We also observe that

$$
\forall x \in(-a, a), \quad \partial_{x} w(x, b)=\frac{1}{2 a}\left(-\Psi(x, b)+k_{a}^{b}\right)+\frac{a-x}{2 a} \partial_{x} \Psi(x, b)<0,
$$

and therefore $y_{0} \neq b$. The remaining case $x_{0} \in(-a, a), y_{0}=0$ is also ruled out because it would imply

$$
0 \geqslant-d \partial_{y} w\left(x_{0}, 0\right)=\mu z\left(x_{0}\right)-\nu w\left(x_{0}, 0\right)=\mu z\left(x_{0}\right)-\nu k_{a}^{b}>0,
$$

where the last inequality follows from the strict lower bound on $z$. We have eventually shown that

$$
\forall x \in[-a, a), \quad \frac{\nu}{\mu} k_{a}^{b}<z(x), \quad \forall y \in[0, b], \quad k_{a}^{b}<w(x, y) .
$$

\section{Uniqueness and monotonicity.}

We prove uniqueness and monotonicity at the same time, using the sliding method. Suppose that $(\widetilde{z}, \widetilde{w})$ is another solution of (7.3) satisfying (7.4). For $\tau>0$, consider the translation $\Omega_{a b}^{\tau}:=(-a-\tau, a-\tau) \times(0, b)$ and the shifted functions

$$
\widetilde{z}_{\tau}(x)=\widetilde{z}(x+\tau), \quad \widetilde{w}_{\tau}(x, y)=\widetilde{w}(x+\tau, y)
$$

defined in $(-a-\tau, a-\tau)$ and $\Omega_{a b}^{\tau}$ respectively. Thus, for $0<\tau<2 a, w$ and $\widetilde{w}_{\tau}$ are simultaneously defined in the rectangle $D_{\tau}:=\Omega_{a b}^{\tau} \cap \Omega_{a b}$ and, likewise, $z$ and $\widetilde{z}_{\tau}$ are defined on $(-a, a-\tau)$. For $\tau \sim 2 a$ the functions on $(-a, a-\tau)$ or in $D_{\tau}$ are close to their boundary values: for $(x, y) \in D_{\tau}$ we have

$z(x) \sim \Phi(-a), \quad \widetilde{z}_{\tau}(x) \sim \frac{\nu}{\mu} k_{a}^{b} \leqslant \Phi(a), \quad w(x, y) \sim \Psi(-a, y), \quad \widetilde{w}_{\tau}(x, y) \sim k_{a}^{b} \leqslant \Psi(a, y)$.

where we have used the definition (7.2) of $k_{a}^{b}$. Recalling that $\Phi$ and $\Psi$ are strictly decreasing in $x$, we get, for $2 a-\tau$ small,

$$
\widetilde{z}_{\tau} \leqslant z \quad \text { in }(-a, a-\tau), \quad \widetilde{w}_{\tau} \leqslant w \quad \text { in } D_{\tau} .
$$

We now start to decrease $\tau>0$ and define

$$
\sigma:=\inf \left\{\tau>0 \quad: \quad \forall \tau^{\prime} \in[\tau, 2 a), \quad \widetilde{z}_{\tau^{\prime}} \leqslant z \text { in }\left(-a, a-\tau^{\prime}\right) \text { and } \widetilde{w}_{\tau^{\prime}} \leqslant w \text { in } D_{\tau^{\prime}}\right\} .
$$

We show that $\sigma>0$ leads to a contradiction. Suppose indeed that $\sigma>0$. Then, by continuity, we still have $\widetilde{z}_{\sigma} \leqslant z$ in $(-a, a-\sigma)$ and $\widetilde{w}_{\sigma} \leqslant w$ in $D_{\sigma}$. We claim that

$$
\widetilde{z}_{\sigma}<z \text { in }[-a, a-\sigma], \quad \widetilde{w}_{\sigma}<w \text { in } \overline{D_{\sigma}} .
$$

The inequalities on the "sides" follow from (7.4) and (7.6):

$$
\begin{aligned}
& \widetilde{z}_{\sigma}(-a)=\widetilde{z}(-a+\sigma) \leqslant \Phi(-a+\sigma)<\Phi(-a)=z(-a), \quad \widetilde{z}_{\sigma}(a-\sigma)=\frac{\nu}{\mu} k_{a}^{b}<z(a-\sigma), \\
& \widetilde{w}_{\sigma}(-a, y) \leqslant \Psi(-a+\sigma, y)<\Psi(-a, y)=w(-a, y), \quad \widetilde{w}_{\sigma}(a-\sigma, y)=k_{a}^{b}<w(a-\sigma, y) .
\end{aligned}
$$


Thus, by the strong maximum principle, $\widetilde{z}_{\sigma}<z$ on the whole $[-a, a-\sigma]$, and $\widetilde{w}_{\sigma}<w$ on $\overline{D_{\sigma}} \backslash(-a, a-\sigma) \times\{0, b\}$. The inequality $\widetilde{w}_{\sigma}<w$ holds when $y=b$ because there the datum is strictly decreasing, as seen in (7.5), and when $y=0$ because $\left(w-\widetilde{w}_{\sigma}\right)\left(x_{0}, 0\right)=0$ leads to the contradiction

$$
0 \geqslant-d \partial_{y}\left(w-\widetilde{w}_{\sigma}\right)\left(x_{0}, 0\right)=\mu\left(z-\widetilde{z}_{\sigma}\right)\left(x_{0}\right)>0 .
$$

Therefore, (7.7) is proved. By continuity, (7.7) holds true with $\sigma$ replaced by $\sigma^{\prime}<\sigma$ close enough to $\sigma$, contradicting the definition of $\sigma$. We have thereby shown that $\sigma=0$, i.e.,

$$
\forall \tau^{\prime} \in[0,2 a), \quad \widetilde{z}_{\tau^{\prime}} \leqslant z \text { in }(-a, a-\tau) \text { and } \widetilde{w}_{\tau^{\prime}} \leqslant w \text { in } D_{\tau} .
$$

Applying these inequalities with $\tau^{\prime}=0$ and switching the roles of $(z, w)$ and $(\widetilde{z}, \widetilde{w})$ we derive the uniqueness result, while applying the inequalities with $(\widetilde{z}, \widetilde{w})=(z, w)$ we deduce that $(z, w)$ is nonincreasing in $z$.

Dependence with respect to the parameters.

On one hand, the continuity of $\Phi, \Psi$ implies that the functions $\Phi_{h}, \Psi_{h}$ are continuous with respect to $h$ (in the $L^{\infty}([-a, a])$ and $L^{\infty}\left(\bar{\Omega}_{a b}\right)$ norms). On the other, the solutions $(\alpha, \beta, \gamma)$ of (6.3) obtained in [9] are continuous with respect to $c \geqslant w^{*}$. It then follows from parabolic estimates and the well-posedness of the Cauchy problem associated with (3.1) that $\Phi, \Psi$ are continuous with respect to $c$ too (always in $L^{\infty}([-a, a])$ and $\left.L^{\infty}\left(\bar{\Omega}_{a b}\right)\right)$. Then, likewise, the fact that the solution of (7.3)-(7.4) is unique implies that it is continuous with respect to $c$ and $h$.

Proof of Theorem 2.2, part (ii). Fix $a, b>0$. Let $(z, w)$ be the solution of (7.3), with $h \in \mathbb{R}$, satisfying (7.4). We have that

$$
\frac{\nu}{\mu} k_{a+h}^{b} \leqslant z(0) \leqslant \Phi(h)
$$

Recalling the definition (7.2) of $k$ and knowing that $(\Phi, \Psi)$ satisfies (2.6), we get

$$
\lim _{h \rightarrow-\infty} \frac{\nu}{\mu} k_{a+h}^{b}=\lim _{h \rightarrow-\infty} \Phi(h)=\frac{\nu}{\mu}, \quad \lim _{h \rightarrow+\infty} k_{a+h}^{b}=\lim _{h \rightarrow+\infty} \Phi(h)=0 .
$$

It then follows from the continuity of $z$ with respect to the parameter $h$ that there exists $h \in \mathbb{R}$ for which $z(0)=\nu /(2 \mu)$. Next, we let $a, b$ vary and, for each pair, we choose $h$ accordingly to this normalization condition. As $a, b \rightarrow+\infty$, by parabolic estimates, the associated $(z, w)$ converge (up to subsequences) locally uniformly to a solution $(\varphi, \psi)$ for the complete travelling front system (2.5), which is nonincreasing in $x$ and satisfies

$$
0 \leqslant \varphi \leqslant \frac{\nu}{\mu}, \quad 0 \leqslant \psi \leqslant 1
$$

together with the normalization condition

$$
\varphi(0)=\frac{\nu}{2 \mu}
$$

Let us show that $(\varphi, \psi)$ fulfils the limiting conditions (2.6). Consider any sequence $\left(x_{n}\right)_{n \in \mathbb{N}}$ diverging to either $-\infty$ or $+\infty$. We have that (up to subsequences) $\varphi\left(x_{n}\right)$ 
converges to a constant $U$ and that $\psi\left(x_{n}, y\right)$ converges to a function $V(y)$ locally uniformly in $y \geqslant 0$. The pair $(U, V)$ is a nonnegative solution of

$$
\left\{\begin{array}{l}
-d V^{\prime \prime}=f(V), \quad y>0 \\
V(0)=\frac{\mu}{\nu} U \\
V^{\prime}(0)=0
\end{array}\right.
$$

with $V \leqslant 1$. Hence, $V$ is concave, from which one readily deduces that either $(U, V) \equiv(0,0)$ or $(U, V) \equiv(\nu / \mu, 1)$ (this can also be obtained from the general Liouville-type result [9, Proposition 4.1]). Consequently, because $\varphi$ is nonincreasing and satisfies (7.8), we eventually infer that $(U, V) \equiv(\nu / \mu, 1)$ if $x_{n} \rightarrow-\infty$ and $(U, V) \equiv(0,0)$ if $x_{n} \rightarrow+\infty$.

Finally, for the monotonicity in $x$, we already know that $\partial_{x} z$ and $\partial_{x} w$ are nonpositive and not identically equal to 0 , due to (2.6). Then, differentiating the system (7.3) with respect to $x$ and applying the usual arguments based on the strong maximum principle and Hopf's lemma, one derives $\partial_{x} z<0$ and $\partial_{x} w<0$.

\section{Existence of travelling fronts in Systems (1.2) and} (1.3)

Let us sketch the adaptations that allow one to extend the previous arguments to the problem which takes into account transport and mortality on the road, hence to prove Theorem 2.3. The tools needed - well posedness and comparison principle for the Cauchy problem associated with (1.2), Liouville-type result and spreading properties - have been derived in [10].

The construction of the strictly monotone supersolution $(\Phi, \Psi)$ follows the same lines of the proof of Lemma 7.1: one starts from an initial datum given by the exponential supersolution $(\bar{\varphi}, \bar{\psi})$ for $(1.2)$ - which is obtained in [10] solving an algebraic system similar to $(6.3)$ - truncated by the steady state, which is now $\left(u_{s}, v_{s}(y)\right)$ instead of $(\nu / \mu, 1)$.

The $y$-dependence of the steady state entails a technical difficulty in the application of the sliding method, and this is where the extra hypothesis on $f$ comes into play. Indeed, for the problem in the truncated domain $\Omega_{a b}$, we need to impose a $y$-dependent datum on the side $x=a$. Namely, we consider

$$
\begin{cases}-c \partial_{x} w-d \Delta w=f(w), & (x, y) \in \Omega_{a b}=(-a, a) \times(0, b), \\ (q-c) \partial_{x} z-D \partial_{x x} z=\nu w-(\mu+\rho) z, & x \in(-a, a), y=0, \\ -d \partial_{y} w=\mu z-\nu w, & x \in(-a, a), y=0, \\ w(x, y)=\frac{a-x}{2 a} \Psi_{h}(x, y)+\frac{a+x}{2 a} k_{a+h}^{b} v_{s}(y), & (x, y) \in \partial \Omega_{a b}, y \neq 0, \\ z(-a)=\Phi_{h}(-a), \quad z(a)=k_{a+h}^{b} u_{s}, & \end{cases}
$$

where $k_{a}^{b}$ is now defined by

$$
k_{a}^{b}:=\min \left\{\frac{\Phi(a)}{u_{s}}, \min _{y \in[0, b]} \frac{\Psi(a, y)}{v_{s}(y)}\right\} .
$$


Observe that $k_{a}^{b}<1$ because $(\Phi, \Psi)$ satisfies (2.8). Then, being $\frac{f(s)}{s}$ decreasing, the pair $k_{a}^{b}\left(u_{s}, v_{s}(y)\right)$ is a subsolution to the above problem, and this is what is needed to make the sliding method work and prove the analogous of Proposition 7.1.

Finally, using the normalization condition $z(0)=u_{s} / 2$, one eventually gets in the limit $a, b \rightarrow+\infty$ a nontrivial solution for the travelling front problem (2.7). In order to show that it fulfils the limiting conditions (2.8), one proceeds as at the end of the proof of Theorem 2.2 part (ii), and concludes applying the Liouville-type result for (2.4), i.e., [10, Proposition 3.1].

The existence proof of travelling wave solutions for System (1.3) follows the same lines, and works precisely because the existence of a 1D steady solution allow to construct a super-solution that has the correct behaviour to the right of the cylinder.

\section{Acknowledgments}

The research leading to these results has received funding from the European Research Council under the European Union's Seventh Framework Programme (FP/2007-2013) / ERC Grant Agreement n. 321186 - ReaDi - "Reaction-Diffusion Equations, Propagation and Modelling" held by Henri Berestycki. This work was also partially supported by the French National Research Agency (ANR), within the project NONLOCAL ANR-14-CE25-0013.

\section{References}

[1] D.G. Aronson, H.F. Weinberger, Multidimensional nonlinear diffusion arising in population genetics, Adv. Math. 30 (1978), 33-76.

[2] R. Barroux, Redouté, le moustique "tigre" est arrivé aux portes de Paris. Le Monde, 17 juillet 2012.

[3] H. Berestycki, G. Chapuisat, Traveling fronts guided by the environment for reactiondiffusion equations, Netw. Heterog. Media 8 (2013), no. 1, 79-114.

[4] H. Berestycki, A.-C. Coulon, J.-M. Roquejoffre, L. Rossi, Speed-up of reactiondiffusion fronts by a line of fast diffusion, Séminaire Laurent Schwartz EDP et applications, (2013-2014), Exp. No. 19, 25 p.

[5] H. Berestycki, A.-C. Coulon, J.-M. Roquejoffre, L. Rossi, The effect of a line with non-local diffusion on Fisher-KPP propagation. Mathematical Models and Methods in Applied Sciences 25 (2015), 2519-2562.

[6] H. Berestycki, F. Hamel, Reaction-diffusion equations and propagation phenomena, Applied Mathematical Sciences, Springer-Verlag, to appear.

[7] H. Berestycki, L. Nirenberg, On the method of moving planes and the sliding method, Boletim Soc. Brasil. de Mat. Nova Ser. 22 (1991), 1-37.

[8] H. Berestycki, L. Nirenberg, Travelling fronts in cylinders. Inst. H. Poincaré, Anal. non Linéaire, 9 (1992), 497-572.

[9] H. Berestycki, J.-M. Roquejoffre, L. Rossi, The influence of a line with fast diffusion on Fisher-KPP propagation. J. Math. Biol. 66 No. 4-5 (2013), 743-766. 
[10] H. Berestycki, J.-M. Roquejoffre, L. Rossi, Fisher-KPP propagation in the presence of a line : further effects, Nonlinearity, 26 (2013), 2623-2640.

[11] H. Berestycki, J.-M. Roquejoffre, L. Rossi, The shape of expansion induced by a line with fast diffusion in Fisher-KPP equations, Comm. Math. Phys., to appear.

[12] T. DE CAmino-BeCK, M.A. Lewis, Invasion with stage-structured coupled map lattices: Application to the spread of scentless chamomile. Ecological Modelling, 220 (2009), 3394-3403.

[13] Canadian Ministry of Agriculture, Government of Saskatchewan, scentless chamomile, http://www.agriculture.gov.sk.ca/Scentless_Chamomile

[14] L. Dietrich, Existence of travelling waves for a reaction-diffusion system with a line of fast diffusion, Appl. Math. Res. Express, 2015.

[15] L. Dietrich, Velocity enhancement of reaction-diffusion fronts by a line of fast diffusion, to appear in Trans. A.M.S.

[16] T. Hillen, K. PAinter, Transport and anisotropic diffusion models for movement in oriented habitats, Lecture Notes in Mathematics, 2071 (2013), 177-222.

[17] Inventaire National Du Patrimoine Naturel, Vespa velutina Lepeletier, 1836, http://inpn.mnhn.fr/espece/cd_nom/433589, 2015.

[18] T. Jung And M. Blaschke. Phytophthora root and collar rot of alders in bavaria: distribution, modes of spread and possible management strategies. Plant Pathology, 53 (2004), 197-208 .

[19] A.N. Kolmogorov, I.G. Petrovskit, N.S. Piskunov, Étude de l'équation de la diffusion avec croissance de la quantité de matière et son application à un problème biologique, Bull. Univ. État Moscou, Sér. Intern. A 1 (1937), 1-26.

[20] O. A. Ladyženskaja, V. A. Solonnikov, and N. N. Ural'Ceva. Linear and quasilinear equations of parabolic type, Translated from the Russian by S. Smith. Translations of Mathematical Monographs, Vol. 23, American Mathematical Society, Providence, R.I., 1967.

[21] H.W. McKenzie, E.H. Merrill, R.J. Spiteri, And M.A. Lewis, How linear features alter predator movement and the functional response. Interface focus 2 (2012), 205-216.

[22] M. H. Protter, H. F. Weinberger., Maximum principles in differential equations. PrenticeHall Inc., Englewood Cliffs, N.J., 1967.

[23] L. Roques, J.-P. Rossi, H. Berestycki, J. Rousselet, J. Garnier, J.- M. RoquejofFre, L. Rossi, S. Soubeyrand, C. Robinet, Modeling the spatio-temporal dynamics of the pine processionary moth. In : Processionary Moths and Climate Change: An Update, Springer 2015 .

[24] A. Siegfried, Itinéraires des contagions, épidémies et idéologies. A. Colin, Paris, 1960. 


\section{Appendix: existence in truncated domain}

The problem under investigation is

$$
\left\{\begin{aligned}
-c \partial_{x} w-d \Delta w & =f(w), & & (x, y) \in \Omega_{a b}=(-a, a) \times(0, b), \\
-c \partial_{x} z-D \partial_{x x} z & =\nu w-\mu z, & & x \in(-a, a), y=0, \\
-d \partial_{y} w & =\mu z-\nu w, & & x \in(-a, a), y=0, \\
w & =\widetilde{w}, & & (x, y) \in \partial \Omega_{a b}, y \neq 0, \\
z & =\widetilde{z}, & & x= \pm a,
\end{aligned}\right.
$$

where the data $\widetilde{w}, \widetilde{z}$ are of class $C^{2}$ and satisfy the compatibility condition

$$
-d \partial_{y} \widetilde{w}( \pm a, 0)=\mu \widetilde{z}( \pm a)-\nu \widetilde{w}( \pm a, 0)
$$

Using an iterative method, we derive an existence result to (A.1). The compatibility condition (A.2) will keep the technicalities due to the corners to a minimum.

Proposition A.1. Assume that the problem (A.1) admits a subsolution $(\underline{z}, \underline{w})$ and a supersolution $(\bar{z}, \bar{w})$ satisfying

$$
\underline{z} \leqslant \widetilde{z} \leqslant \bar{z} \quad \text { on }\{ \pm a\}, \quad \underline{w} \leqslant \widetilde{w} \leqslant \bar{w} \quad \text { on }\left(\partial \Omega_{a b}\right) \backslash((a, b) \times\{0\}) .
$$

Then, there exists a solution $(z, w)$ satisfying

$$
\underline{z} \leqslant z \leqslant \bar{z}, \quad \underline{w} \leqslant w \leqslant \bar{w} .
$$

Proof. With split the coupled system into two separate problems. Set $z_{0}:=\bar{z}$ and $w_{0}:=\bar{w}$. Consider the problem for $z$ :

$$
\left\{\begin{aligned}
-c \partial_{x} z-D \partial_{x x} z & =\nu w(x, 0)-\mu z, & & x \in(-a, a), \\
z & =\widetilde{z}, & & x= \pm a
\end{aligned}\right.
$$

with $w \equiv w_{0}$. The functions $\underline{z}$ and $z_{0}=\bar{z}$ are a sub and a supersolution for this problem. Then, by standard arguments, there exists a solution $z_{1}$ satisfying $\underline{z} \leqslant z_{1} \leqslant$ $z_{0}$. Next, consider the problem for $w$ :

$$
\left\{\begin{aligned}
-c \partial_{x} w-d \Delta w & =f(w), & & (x, y) \in \Omega_{a b}, \\
-d \partial_{y} w & =\mu z-\nu w, & & x \in(-a, a), y=0 \\
w & =\widetilde{w}, & & (x, y) \in \partial \Omega_{a b}, y \neq 0
\end{aligned}\right.
$$

with $z \equiv z_{1}$. The functions $\underline{w}$ and $w_{0}=\bar{w}$ are a sub and a supersolution of this problem and then, thanks to the compatibility condition (A.2), there exists a solution $\underline{w} \leqslant w_{1} \leqslant w_{0}$.

We iterate the above procedure obtaining two nonincreasing sequences $\left(z_{n}\right)_{n \in \mathbb{N}}$ and $\left(w_{n}\right)_{n \in \mathbb{N}}$ such that

$$
\forall n \in \mathbb{N}, \quad \underline{z} \leqslant z_{n} \leqslant z_{n-1} \quad \text { and } \quad \underline{w} \leqslant w_{n} \leqslant w_{n-1},
$$


with $z_{n}$ solution of (A.3) with $w=w_{n-1}$, and $w_{n}$ solution of (A.4) with $z=z_{n}$. Notice, indeed, that if the above properties hold for some $n$, then the problem (A.3) with $w=w_{n}$ admits $\underline{z} \leqslant z_{n}$ as sub and supersolution, which allows us to define $z_{n+1}$; then, (A.4) with $z=z_{n+1}$ admits $\underline{w} \leqslant w_{n}$ as sub and supersolution and thus we can define $w_{n+1}$.

The desired solution $(z, w)$ is the uniform limit of $\left(\left(z_{n}, w_{n}\right)\right)_{n \in \mathbb{N}}$.

Systems (1.2) and (1.3) are treated along the same lines. 